Carnets de géographes

GÉOGRAPHES

$7 \mid 2014$

Les espaces de l'entre-deux

\title{
La présence des petits bâtiments en ruine dans les Alpes-de-Haute-Provence
}

Construction diachronique d'un paysage de l'entre-deux

Quentin Morcrette

\section{OpenEdition}

\section{Journals}

Édition électronique

URL : http://journals.openedition.org/cdg/538

DOI : $10.4000 /$ cdg. 538

ISSN : 2107-7266

\section{Éditeur}

UMR 245 - CESSMA

\section{Référence électronique}

Quentin Morcrette, «La présence des petits bâtiments en ruine dans les Alpes-de-Haute-Provence »,

Carnets de géographes [En ligne], 7 | 2014, mis en ligne le 01 décembre 2014, consulté le 10 décembre 2020. URL : http://journals.openedition.org/cdg/538 ; DOI : https://doi.org/10.4000/cdg.538

\section{(c) $(1) \&$}

La revue Carnets de géographes est mise à disposition selon les termes de la Licence Creative Commons Attribution - Pas d'Utilisation Commerciale - Pas de Modification 4.0 International. 


\title{
La présence des petits bâtiments en ruine dans les Alpes-de-Haute-Provence Construction diachronique d'un paysage de l'entre-deux
}

QUENTIN MORCRETTE Communauté Académique de Recherche, Rhône-Alpes / Cluster 5 IRG - UMR 5600 / EVS. Université Lyon II

Géographe

quentin.morcrette@univ-lyon2.fr

\section{Résumé}

De nombreux petits bâtiments en ruine (bergeries, fermes, habitations...) émaillent le territoire des Alpes du Sud. Leur présence n'est pas neutre et s'explique par l'importante déprise connue par la région durant le $19^{\mathrm{ème}}$ et le $20^{\mathrm{ème}}$ siècle. Cette étude s'attache à souligner le rôle de ces ruines dans le paysage de quelques vallées des Alpes-de-HauteProvence. A notre sens, celles-ci représentent à proprement parler le lieu de l'entre-deux, à la fois entre l'homme et la nature, entre le passé et le présent, entre ce qui est productif et ce qui ne l'est pas. Pour les habitants de la région par ailleurs, ces espaces de marges interrogent et marquent, comme des signes du passé et d'un territoire alors organisé différemment.

\begin{abstract}
Many small ruined building (sheepfolds, farms, houses...) are visible in Southern French Alps' landscape. Their presence is not neutral. It may be explained by the important rural exodus that struck the area during the $19^{\text {th }}$ and $20^{\text {th }}$ centuries. This study focuses on the role of these ruins in the landscape of several valleys in the Alpes-de-Haute-Provence. From our point of view, it might be described as the place of the in-between, between human and nature, between past and present, between what is productive and what is lost. It participates in the creation of a space of the in-between. Cartographical analysis and field work showed how this ruins are distributed in the area and proved that they are considered as important signs for the inhabitants of the region, some consider it as interesting landmarks, on the contrary, others would rather not see it here.
\end{abstract}




\section{Introduction}

Un peu à l'écart, ou bien peut-être un peu dans l'entre-deux moi-même, lors du choix de mon sujet de recherche en master, j'avais choisi d'étudier, sans le nommer ainsi, ce lieu de l'entre-deux qu'est la ruine. Entre-deux car à la frontière du bâti et non bâti, de la pierre et du végétal, de "l'ceuvre humaine et [de]celle de la nature", pour reprendre les mots de Georges Simmel (Simmel, 1912).

Un peu à l'écart, dans la mesure où mon angle d'approche ne semblait pas être des plus en vogue parmi les géographes français et il me semble moi-même avoir finalement été souvent à l'entre-deux (posture bien souvent fructueuse). J'étais alors intéressé par les petits vestiges en milieu rural ; des fermes, des maisons individuelles, des cabanes, voire des villages entiers abandonnés, dispersés sur le territoire; des ruines qui ne s'apparentent pas à celles qui forment le "grand patrimoine ". Mon terrain d'étude s'organisait autour de quelques-unes des vallées des Alpes-de-Haute-Provence, à l'Est de l'axe durancien, bordé par le plateau de Valensole au Sud et le massif du Queyras au Nord. C'est un territoire rural, polarisé par l'axe durancien et la préfecture de Digne-les-Bains. Le relief est ici plus affirmé et capricieux que dans la " basse-Provence " mais n'a pas encore l'ampleur et l'ouverture des massifs plus centraux.

Frappé par la présence des nombreuses ruines dans le paysage, qui sonnait en fait comme une grande absence, je me rappelais cette définition du Dictionnaire des Idées Reçues: "Ruines: Font rêver et donnent de la poésie à un paysage " (Flaubert, 1881 : 550), et je voulais tenter de répondre, de manière plus objective, à une question que l'on pourrait résumer simplement : " qu'est-ce que cela fait que la ruine soit là » ? Comment est-ce que I'on peut expliquer cela historiquement ? Comment ces vestiges sont perçus par les acteurs des territoires étudiés ? Parce qu'elle était axée sur un type objet inscrit spatialement et historiquement, cette étude exigeait d'englober des champs variés, allant de l'histoire de la région aux dynamiques touristiques actuelles, en passant par de l'analyse spatiale et des entretiens pour comprendre la perception des habitants. II s'agissait d'expliquer dans un ordre logique, à la fois les raisons de la présence des ruines sur le territoire et la manifestation de celle-ci.

Ce que je souhaite faire ici c'est revenir sur quelques-unes des observations que j'ai eu l'occasion de faire et qui m'ont amené à penser que ce bâtiment en ruine qui se dresse devant nos yeux est précisément le lieu de l'entre-deux. Aussi, je rappellerai dans un premier temps la façon dont j'ai abordé ce terrain et les méthodes mobilisées ; puis je m'attacherai à questionner la nature de la ruine et montrerai comment elle s'insère dans des logiques paysagères particulières à la fois d'un point de vue théorique et pour les acteurs rencontrés. 


\section{Le terrain et l'objet}

\section{Du recensement des vestiges sur les cartes à la confrontation avec le terrain}

Ma découverte de ce territoire s'est faite en même temps que la découverte de mon sujet d'étude, par une approche avant tout cartographique.

La multitude de ruines recensées sur les cartes de l'IGN m'a tout d'abord interrogée, j'ai commencé à les dénombrer sur les cartes, à coller des gommettes sur chaque figuré, de manière très sommaire. J'ai continué cette démarche par informatique, pour créer un système d'information géographique avec lequel j'allais pouvoir faire de l'analyse spatiale en comptabilisant quelques 1256 vestiges répartis sur 68 communes. Le service national de cartographie français considère comme ruine toute " construction ancienne abandonnée et inhabitable, dont le toit s'est effondré ou a disparu, et généralement envahie par la végétation ${ }^{1}$. Celles-ci sont facilement repérables du fait $d^{\prime}$ un symbole conventionnel, qui se double parfois d'une désignation "Rnes" si ces vestiges sont considérés comme importants ou présentent un intérêt particulier. Après ce "recensement ", aucune véritable logique dans la répartition des vestiges ne s'est dégagée; les ruines que je cherchais étaient réparties assez également sur le territoire d'étude. Un élément me frappait tout de même, les parties hautes, que se soit à l'échelle de la zone d'étude ou bien des communes possédaient plus de vestiges que les parties basses.

La confrontation avec le terrain m'a amené à réviser certaines informations puisque, une fois sur place, force fut de reconnaitre que les cartes n'indiquaient pas le territoire; qu'elles avaient fixé sur le papier l'état de celui-ci à un instant « $T$ » et que les ruines avaient évolué rapidement, nous y reviendrons. Ainsi me suis-je plusieurs fois retrouvé à l'emplacement exact d'une ruine indiquée sur la carte pour y trouver, soit un champ vide, soit un bâtiment nouvellement construit ou réhabilité.

\section{Aspect de la ruine dans les Alpes de Haute-Provence}

Mais, finalement, plus que la présence ou l'absence d'une ruine, il faut reconnaitre la diversité des types de vestiges auxquels j'ai été confronté. En effet, certaines des ruines que j'ai pu reconnaitre, visiblement abandonnées depuis peu présentaient encore des murs entiers, voire des pans de toits, tandis que d'autres dessinaient à peine sur le sol l'empreinte des fondations de l'ancien bâtiment. Les photographies suivantes illustrent cette diversité.

\footnotetext{
${ }^{1}$ Th. Gerbaud, communication personnelle.
} 
Figure 1 : Une ruine peu dégradée

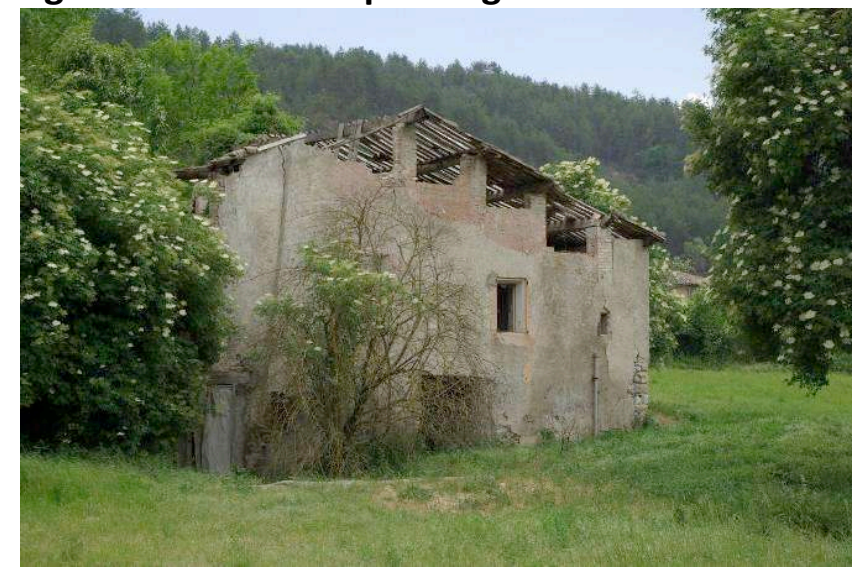

Source : Quentin Morcrette, juin 2010.

Cette habitation, visiblement abandonnée depuis peu, dont les murs sont encore recouverts de crépis, était largement visible depuis le bord de la départementale qui remonte la vallée de la Bléone, sur la route de Digne-les-Bains.

\section{Figure 2 : Une ruine à pan de murs}



Source : Quentin Morcrette, avril 2010.

Ce second vestige est dans un état de dégradation plus avancé. La situation, légèrement isolée, sur un plateau, permet de penser que ce bâtiment était lié à une exploitation agricole. 
Figure 3 : Une ruine presque disparue

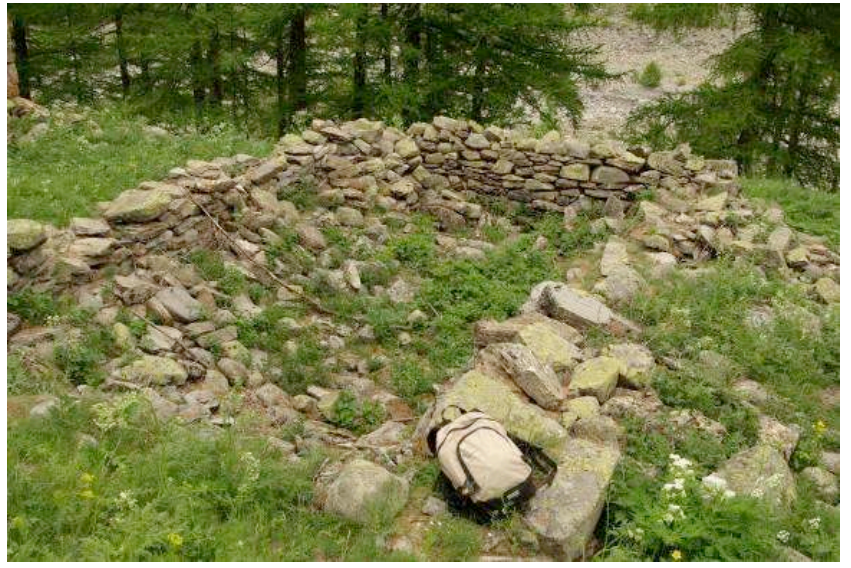

Source : Quentin Morcrette, juin 2010.

Enfin, on peut voir ici ce qu'il reste d'une cabane de berger sur les hautes terres de l'Ubaye ; ce vestige est à peine visible dans le paysage de rocaille et de forêt d'altitude.

A la suite de mes observations de terrain, j'ai regroupé les vestiges en une typologie comprenant sept types de ruines, de celles très bien conservées à celles dont il ne reste quasiment plus la marque au sol (en somme, cette typologie se basait sur leur degré d'avancement dans la ruine). Le bâtiment commence logiquement par se dégrader dans les parties hautes, le toit est perdu d'abord, puis les sommets des murs se dégradent de manière assez inégale, des pans entiers de murs partent ensuite, avant qu'il n'y ait plus, finalement, que la forme générale du bâtiment inscrite sur le sol. Cette diversité des ruines est due à l'ancienneté de l'abandon; mais pour ce qui est de la forme et de la taille des ruines, c'est aussi lié à la diversité des types de bâtiments, des villages entiers ont en effet été abandonnés dans la région (Roncayolo, 1965), mais aussi des fermes isolés ou encore des bergeries. De ces deux éléments, c'est le premier finalement qui influe le plus sur le rôle des ruines dans la constitution d'un paysage de l'entre-deux, nous y reviendrons. II nous faut tout d'abord revenir sur un aspect qui place ces petites ruines rurales dans l'entre-deux : les travaux de géographes sur des thèmes similaires.

\section{Un thème en marge}

Les études sur les ruines ou les vestiges ruraux sont peu nombreuses chez les géographes. En 1911 pourtant, Vidal de la Blache rappelait : « Il y a, comme disait Ratzel, une géographie des ruines, et la persistance dont elles font preuve dans les contrées de la pierre et du mortier est en elle-même un fait géographique » (Vidal de la Blache, 1911).

Aujourd'hui, géographie urbaine comme rurale traitent de thèmes voisins, la première en appelant friche ce qui est en ruine, la seconde en traitant des dynamiques d'enfrichement mais en ignorant alors la question du bâti.

Le terme de "friches" est en effet largement utilisé pour désigner les bâtiments abandonnés en milieu urbain ou périurbain. Les études sur ce thème privilégient alors souvent une approche aménagiste ou bien analysent les processus de réhabilitation et d'appropriation sociale de ces espaces mixtes (Gravari-Barbas, 2010 ; Veschambre, 2008). 
Mais, dans sa première acceptation, ce terme désigne " un champ, un espace agricole récemment abandonné et laissé à la végétation spontanée " (Lussault, Lévy, 2003). De fait, la géographie rurale s'est emparée du sujet et nombre d'études évoquent ainsi l'entre-deux à travers l'étude du processus de "l'enfrichement " (Equipe de recherche dynamique des paysage et aménagement régional, 1991; Fabre, 2006). Ce terme désigne alors la multiplication des espaces intermédiaires entre le champ cultivé et la forêt, l'abandon d'un champ se traduisant par la croissance de la friche sur ce dernier, donc d'une zone de végétation mixte. Le processus continuant, c'est alors souvent une zone forestière qui remplace les anciens paysages ouverts, c'est ce que l'on nomme la fermeture des paysages (Deuffic, 2005; Le Floch, 2005; Moustier, 2006).Au sein de ces études, qui traitent finalement de l'entre deux, la question du bâti rural semble pourtant souvent négligée.

La figure suivante (Bazin, 1986), met en lumière le phénomène bien connu du l'abandon des parcours ainsi que des cultures sur les versants; qui a généré l'enfrichement croissant des paysages de montagnes tout au long du $20^{\text {ème }}$ siècle. II souligne parallèlement la concentration de l'agriculture dans les vallées. Mais, alors même que l'auteur décrit un changement de système générant la présence d'une ruine dans le paysage, le bâtiment qui est représenté sur le haut du versant pour le schéma de la situation au $19^{\text {ème }}$ siècle a disparu pour le schéma concernant le $20^{\text {ème }}$ siècle.

\section{Figure 4 : Evolution de l'utilisation du sol dans une vallée des Alpes du Sud}



La même vallée en 1980



Source : Gilles Bazin (1986, in Quelles perspectives pour les agricultures montagnardes ? Exemples du Massif Central et des Alpes du Sud) 
Or, le bâtiment ne disparait pas instantanément s'il est abandonné, il devient d'abord une ruine, symbole de la ruine d'un système disparu; " s'il y a trace, c'est qu'il y a eu perte ", pour reprendre les mots de Sylvie Lacroix (Lacroix, 2007)

\section{Explication de l'apparition des ruines}

La région se caractérise aujourd'hui par un peuplement très faible, un habitat assez hétérogène regroupé le long des axes de communication et des vallées, avec quelques bourgs d'altitude sur des plateaux ouverts ou des versants évasés. Mais cela n'a pas toujours été vrai et ces montagnes étaient très peuplées au $19^{\text {ème }}$ siècle. Les nombreuses ruines isolées dans les anciennes cultures, ou perchées à flanc de montagne, trouvent ainsi leur origine dans l'histoire récente du territoire étudié. Pour citer quelques chiffres, on peut rappeler que le peuplement était ici à son optimum vers 1850 ; le nombre d'habitants est ainsi passé, dans le département des Basses-Alpes, de 159000 en 1836 à 84000 en 1954, pour remonter lentement depuis.

Epuisement d'un sol maigre surexploité durant le $19^{\text {ème }}$ siècle, concurrence entre l'élevage local et les troupeaux transhumant, réduction de l'isolat économique avec le développement des transports, autant d'éléments qui ont précipité la ruine du système agro-pastoral local (Merlin, 1969), et ont poussé la population locale, en grande majorité paysanne, à s'exiler à partir de la fin du $19^{\text {ème }}$ siècle (comme ces nombreux barcelonettes qui tentèrent l'aventure au Mexique...). Cette baisse de la population toucha l'ensemble du territoire étudié, et dura jusqu'aux années 1975. Cela prit parfois la forme d'une véritable désertion; ainsi, parmi d'autres, les villages de Blieux et de Majastres, situés au Sud de Digne-les-Bains, perdirent respectivement 94 et $99 \%$ de leur population entre 1841 et 2006.

La carte ci-dessous, qui concerne la zone Sud-Est du territoire que nous avons étudié, permet d'appréhender la très forte baisse de la population dans de nombreuses communes depuis un siècle et demi. 
Figure 5: Evolution de la population et présence de ruines dans le Sud-Est du territoire étudié, entre 1841 et 2006

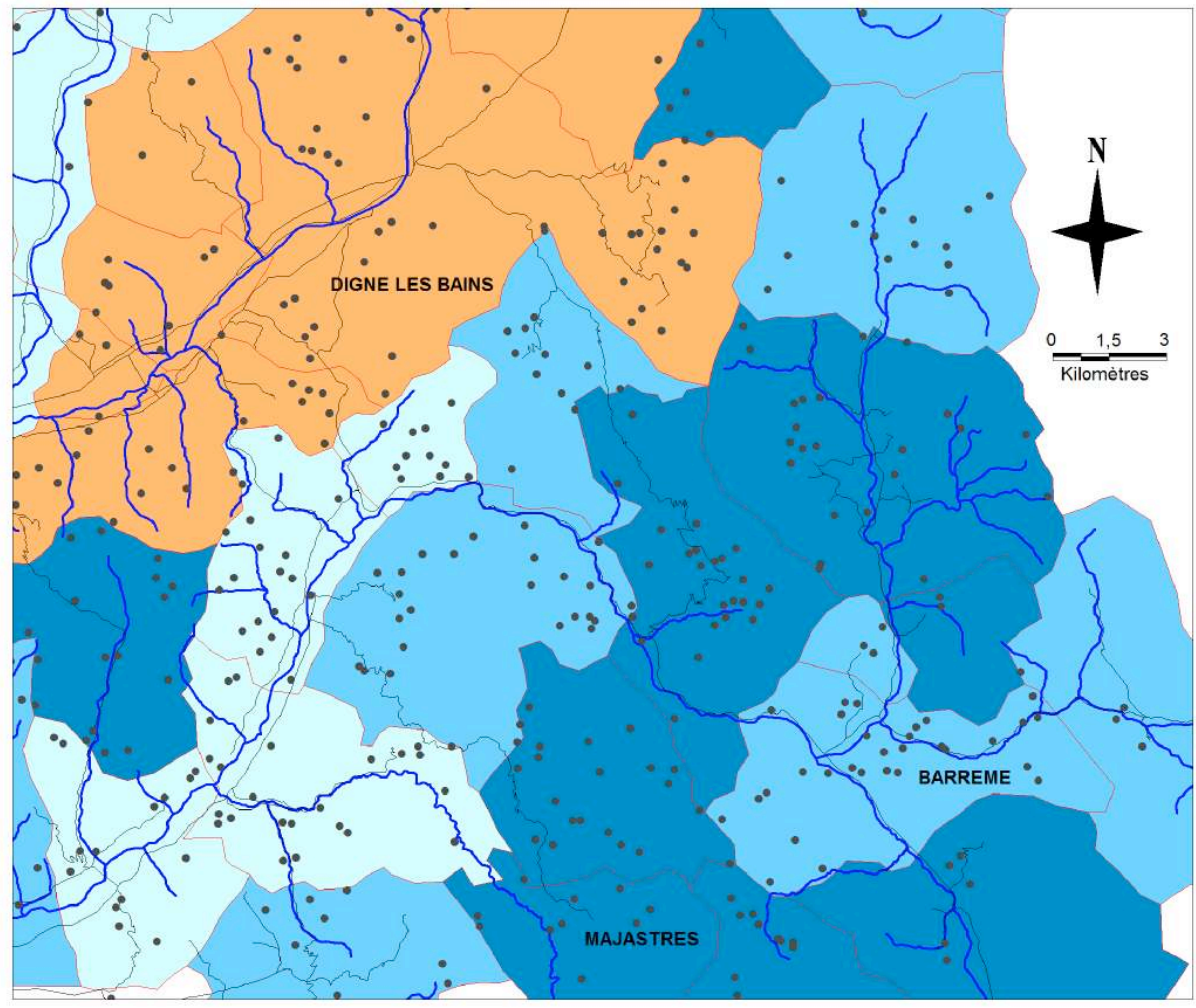

\section{De +25 à $+290 \%$ (Dignes-les-Bains) \\ De -50 à $0 \%$ \\ De -75 à $-50 \%$ \\ De $-99,25$ (Majastres) à $-75 \%$}

Evolution relative de la population entre 1841 et 2006

\section{Limites communales}

Quentin Morcrette, juillet 2010.

\section{$\underline{\text { Vestiges recensés }}$}

$\underline{\text { Cours d'eau }}$

On constate que seule la commune de Digne-les-Bains n'a pas perdu de population entre 1841 et 2006. Dans les autres zones, malgré la stabilité voire la hausse de la population actuellement en cours, la population est toujours inférieure à ce qu'elle était au $19^{\text {ème }}$ siècle. Ceci est particulièrement vrai dans le "bloc de Majastres" (autour de la commune éponyme, dans l'angle Sud-Est ici), massif très isolé et compact (le village de Majastres luimême n'est relié à la vallée de l'Asse que par une route, en très mauvais état, se terminant en cul de sac après 15 kilomètres et le passage de gorges spectaculaires).

C'est le départ de la population qui a engendré la présence des ruines, les bâtiments se retrouvant, de ce fait, inoccupés. Cependant, le travail du géographe est aussi de manier les échelles et, force est alors de constater que se dégagent aussi des logiques locales; sous l'effet des changements systémiques les structures de peuplement et de l'économie ont été modifiées. 
Dans les zones de moyenne montagne, l'extension du domaine cultivé se faisait souvent à partir d'un abri isolé progressivement transformé en ferme. Ce sont les marges extrêmes qui ont été défrichées en dernier lieu, lors de la hausse de la population, par une logique de " point de conquête " plus que de "front pionnier ", lors de la baisse de la population, elles ont aussi été les premières à être abandonnées. De plus, comme le dit $\mathrm{M}$. Roncayolo :

"La désertion des terres lointaines, moins fertiles, moins bien situées n'est souvent que le négatif d'un autre phénomène: le développement d'une agriculture plus intensive, plus exigeante en main-d'œuvre dans les zones de grande circulation, dans l'arrière-pays de ces villes, dont le poids s'affirme au 20ème siècle » (Roncayolo, $1965: 45$ ).

C'est donc logiquement sur les meilleures terres que l'on trouverait le moins de désertion et le moins de vestiges. L'on comprend ici que les zones qui ne font pas partie d'un axe de circulation majeur, c'est-à-dire d'une vallée importance, doivent posséder plus de ruines. D'où la grande irrégularité des taux de corrélation entre baisse de la population d'une commune et nombre de ruines sur cette dernière, du fait de l'étagement de nombreuses communes. Les communes très étagées peuvent avoir perdu moins de population que des communes d'altitude et pourtant posséder plus de ruines sur leur partie haute.

Ainsi, départ de la population et changements socio-économiques ont généré l'abandon des bâtiments agricoles, fermes et bergeries isolées; ceux-ci, aujourd'hui en ruine au sein des anciens champs exploités, génère par leur présence ce que l’on peut appeler un paysage de l'entre-deux.

\section{La ruine dans le paysage, de l'entre-deux théorique à l'entre-deux perçu}

\section{Définir la ruine comme un triple entre-deux}

Si l'on définit l'entre-deux comme un état intermédiaire, entre-deux choses, donc dans l'intervalle, et dans la transition (l'entre-deux n'est pas le seuil, qui suppose la rupture); alors, s'intéresser à ce que l'on peut appeler la " petite » ruine rurale, c'est s'attacher à un espace triplement dans l'entre-deux. En effet, elle est à la jonction entre ce qui est dans et hors du territoire (entendu comme espace productif), elle est inoccupée, en marge, mais toujours susceptible d'être réhabilitée et donc réintégrée dans des circuits productifs. Parce qu'elle est, dans le paysage actuel, une trace de systèmes révolus, elle est à l'interface entre le passé et le présent et est un entre-deux temporel. Enfin, la ruine est le lieu de l'entre deux en tant qu'interface entre la nature et la société à l'endroit même où le lierre mord la pierre.

Cet entre-deux ne peut exister que tant que la ruine est ruine et cessera donc d'être dès lors qu'elle sera réhabilitée, ou bien qu'elle aura disparu avec le temps. C'est pourquoi mon étude ne s'est pas attachée à un espace de l'entre-deux institutionnalisé (comme les bâtiments industriels réhabilités, à l'image de la Friche de la Belle de Mai à Marseille). Le motif à l'origine de cette étude s'organisait au contraire autour d'un type de lieu ; finalement défini comme dans l'interface, l'intermède, une zone de marge au cœur des 
préoccupations car signifiante dans le paysage. Donc précisément ce que l'on peut nommer un entre-deux.

L'évolution d'un objet qui amène à la ruine peut être pensée selon une temporalité qui définit son " existence » : I'arrivée d'une population, la construction du bâtiment, le départ de la population, la "ruinification" (le processus pour lequel j'ai défini une typologie suivant l'état de dégradation des ruines) et la disparition. Le dernier état, logique, de la ruine est donc sa destruction. La ruine est donc toujours à la limite de la ruine et « travaille elle-même à sa propre perte " (Riegl, 1903). Cet objet fugitif, sans cesse en transition, se refuse à la stabilité, donc, à la classification, puisque sitôt décrit, bientôt disparu du paysage. La ruinification est en somme la transformation d'un objet bien visible en un objet moins visible dans le paysage. Très concrètement, une maison au crépi rose devient un bâtiment gris-vert, aux contours incertains, faiblement visible. L'objet est ainsi, paradoxalement, plus absent lorsqu'il existe que lorsqu'il n'existe pas. Habitation debout et ruine tombée sont un seul et même objet transformé, qui prend donc un nouveau sens dans le paysage.

\section{La ruine, à l'entre-deux de l'existence dans le paysage}

$\mathrm{Si}$, longtemps, dans la tradition vidalienne, décrire le paysage revenait à distinguer les différentes unités constituant un "pays" (par exemple ager, saltus et sylva méditerranéens), la notion s'est renouvelée en s'attachant au paysage " réel », c'est-à-dire celui perçu par l'utilisateur de l'espace (Lacoste, 1977). Cela définit une géographie du paysage visible dans un espace en trois dimensions. Autrement dit, tous les éléments d'un paysage ne sont pas également visibles sur le terrain, cela revient à ne plus le considérer comme un tout homogène et une composante d'un paysage peut en cacher une autre à l'œil du passant.

Sur le terrain, en croisant plusieurs facteurs, tels la taille des vestiges, leur état de dégradation (comme vu précédemment), leur degré de recouvrement par la végétation, leur proximité avec un axe de communication... j'ai abouti à une classification des ruines définissant leur visibilité dans le paysage. Au terme de ce travail, j'ai pu me rendre compte que la ruine est toujours susceptible de ne plus « exister » pour l'observateur, c'est-à-dire de ne plus être visible dans le paysage. La comparaison de deux clichés du même vestige à deux périodes différentes, permet de rendre compte de l'importance des éléments de végétation dans l'impact paysager des vestiges ruraux: 
Figure 6 : Un vestige envahi par la végétation sur la commune de Faucon du Caire

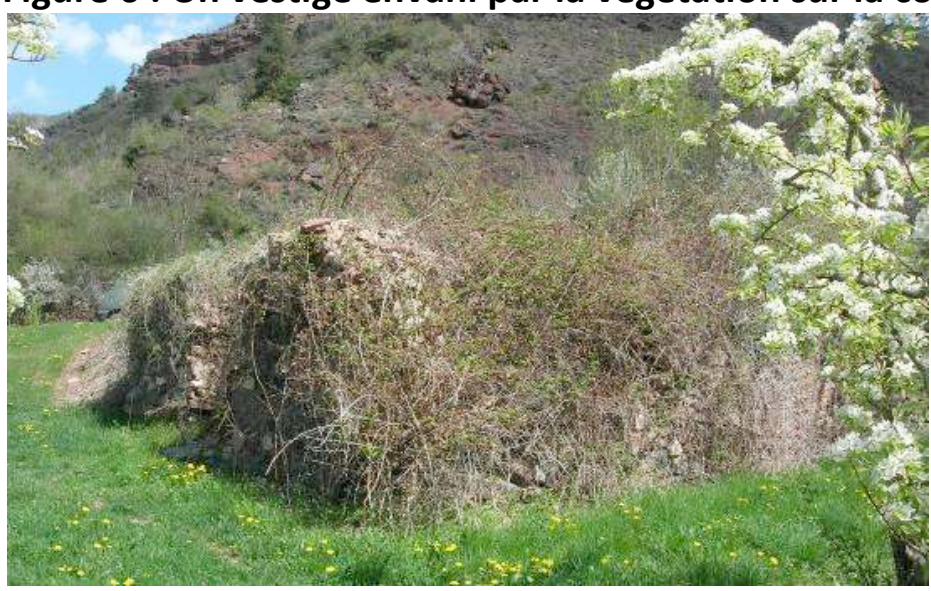

Source : Quentin Morcrette (juin 2010).

Figure 7 : Le même édifice libre de végétation

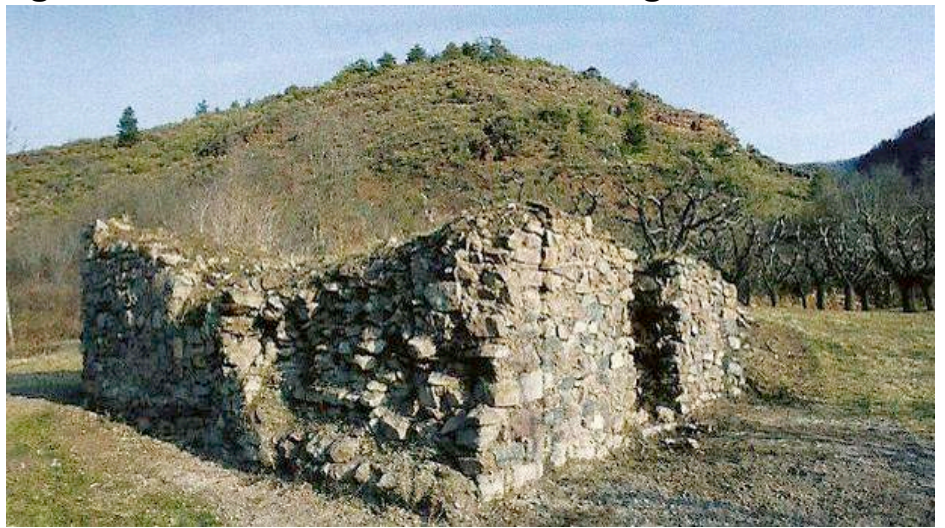

Source : Gérald Lucas (2008, in Les Hautes Terres de Provence, itinérances médiévales)

Sur la photographie de la figure 6, la structure du bâtiment est à peine visible, une dense végétation de ronciers recouvre totalement ce qu'il en reste. Sur la deuxième photographie, les ronciers ont été enlevés, les pierres sont à nu et le bâtiment, bien que très délabré, est ainsi tout de même bien visible dans le paysage.

Il est intéressant de noter ce paradoxe : on ne peut, à la fois, voir dans le paysage la ruine et la friche, ou, du moins, seulement jusqu'à un certain point. La première disparait lorsque la végétation - objet en constante évolution - croit à tel point que la ruine - objet en constante involution par définition -est submergée. Les traces de l'abandon disparaissent ainsi lorsque les effets de l'abandon se manifestent.

Si tant est que "l'existence " d'une ruine dans le paysage n'aie de sens que parce qu'un observateur la perçoit, alors l'existence même de la ruine est modulée par les particularités du lieu dans lequel elle s'insère, par son site et sa situation. Elle apparait, à travers cette analyse, comme un élément fragile des structures du paysage - à l'image de la fragilité qu'elle révèle par son état même. Ceci illustre ce que l'on pourrait appeler une " paysagéité temporelle " (pour le terme de paysagéité, voir Gendrat-Claudel A., 2007) de la ruine. C'està-dire que la structure spatiale de la ruine, donc sa visibilité, est soumise à l'action du temps. Ceci selon deux formes: le temps linéaire qui multiplie la végétation sur la ruine, et fait 
tomber les pierres, mais aussi le temps cyclique qui fait repousser la végétation en été. Ces vestiges envahis par la végétation sont ainsi du ressort d'un micro-paysage, dont la perception ponctuelle, et surtout répétée, par les utilisateurs de l'espace, participe de la création d'un espace vécu.

\section{Un entre-deux perceptible par tous}

Signe d'un temps, d'un système révolu qui apparait encore aujourd'hui à l'observateur, la ruine apparait en ce sens comme une "épilepsie du paysage " (Curnier, 2009). C'est-à-dire un perpétuel rappel de ce que ce paysage était, superposé sur ce qu'il est aujourd'hui.

Le paysage actuel est un héritage où les strates successives se recouvrent et se cachent. L'ensemble alors formé par cette stratification n'est pas égal à la dernière structure visible, mais est la somme de tous les états qui se sont succédé. En ce sens, le paysage est souvent comparé à un palimpseste (Brunet, 1995 ; Bertrand 2002), ce parchemin que l'on grattait pour effacer des textes anciens afin de pouvoir à nouveau écrire dessus. Il est parfois possible aujourd'hui de déchiffrer ces textes qui ont été mal grattés ; ceci se fait souvent à l'aide de procédés techniques comme les ultra-violets et un savoir-faire complexe est toujours nécessaire à ce déchiffrement. Le paysage se révèle à son tour comme un palimpseste mal gratté pour ceux qui s'y penchent et prennent le temps de le comprendre, mais il faut alors avoir les outils pour le déchiffrer. Si la ruine constitue à mon sens plus qu'un palimpseste dans le paysage, c'est qu'elle est visible par tous, et surtout compréhensible par chacun comme la marque des évolutions des structures spatiales tandis que d'autres éléments hérités sont nettement moins visibles. Tout le monde comprend ce que signifie la présence d'une ruine. Mais tout le monde ne " voit " pas l'ancien champ qui se cache sous la forêt. En somme, pour reprendre la distinction opérée par Roger Brunet (Brunet, 1995), plus que d'être des " signes pour le chercheur », ces ruines sont des "signes pour l'usager ».

Mais finalement, c'est aux gens du lieu que l'on doit l'existence de cet entre-deux, car, c'est dans le regard des habitants que l'on peut le connaitre. Lors de mon séjour sur le terrain, j'ai rencontré agriculteurs, élus, retraités, anciens habitants ou nouveaux venus, pour comprendre la manière dont ils percevaient ces vestiges. Nos entretiens ont révélé la proximité des acteurs avec ces espaces de marges. Pour les plus anciens, cela peut avoir à faire avec une histoire personnelle ou familiale, qui donne à la présence de ces vestiges une signification particulière. On retrouve en somme l'idée de la ruine comme palimpseste, comme un entre-deux entre passé et présent. Ainsi, R. ${ }^{2}$, dont la famille est ici depuis des générations, évoque-t-il son rapport aux vestiges :

"C'est pour ça que je préfère avoir une ruine rasée que d'avoir des pans de murs... Parce que, au point de vue aspect, au point de vue intégration dans le paysage, une ruine c'est pas beau quoi ! C'est complètement négatif. Parce que ça rappelle une période où malheureusement il y avait un exode important, et... et il aurait pas fallu le connaitre ça, mais enfin... C'est fait c'est fait quoi ! J'aime pas voir ça !».

\footnotetext{
${ }^{2}$ Par soucis d'anonymat, les noms des personnes interrogées ont été remplacés par des initiales arbitraires.
} 
Monsieur M. est agriculteur dans une petite vallée, il a connu certaines de ces maisons habitées, son père et son grand père avant lui possédaient par ailleurs des bâtiments agricoles aujourd'hui totalement à l'abandon. "Ces ruines [...] ça n'a aucune valeur, et en principe un patrimoine, ça a de la valeur. Ces ruines, à part une valeur sentimentale, c'est tout ce qu'elles ont. Mais tout ça, ça se perd au fil du temps".

A chaque entretien, les interlocuteurs sont capables de citer précisément les vestiges autours du village, ils notent des évolutions, des cabanes qui ont disparu, des granges qui ont été restaurées, etc. En somme, ils ont l'expérience du territoire et connaissent l'emplacement des ruines. Cette connaissance s'associe à la mémoire du lieu, pour faire de la ruine cet entre-deux qui embarrasse, autant la mémoire que le champ cultivé. On ne sait qu'en faire, la plupart du temps, on ne se risquerait pas à la détruire, mais on ne peut pas non plus la restaurer. Elle reste ainsi, dans le balancement incertain entre présence et disparition.

Associés au souvenir d'une autre organisation de la région, les vestiges rappellent l'important déclin démographique. En témoigne ce mot, trouvé dans un livre d'or, placé dans le refuge ouvert au public du village ruiné du Poil (sur la commune de Senez) :

Figure 8 : Extrait du livre d'or du refuge du Poil

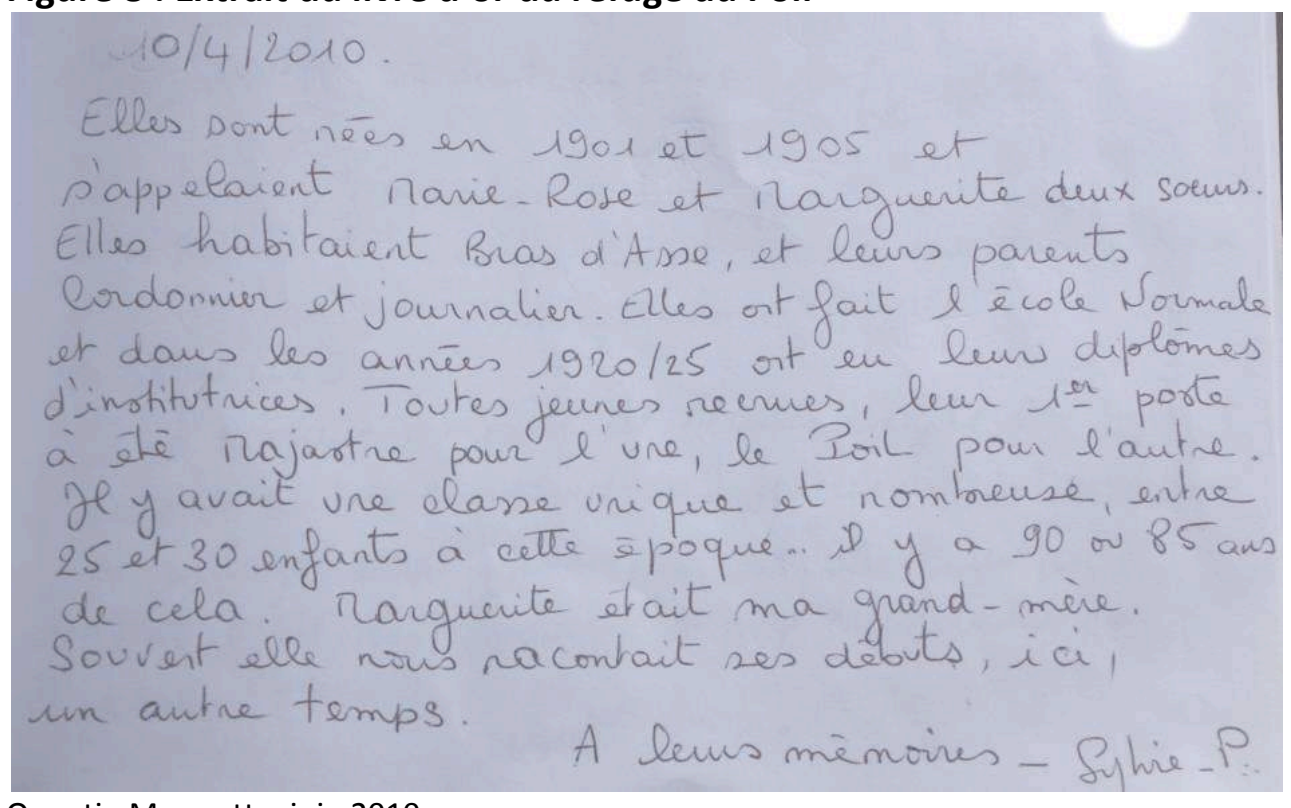

Quentin Morcrette, juin 2010.

Ce que nous rencontrons ici, c'est la ruine témoin, dont la présence nous parle de l'absence. Certains bâtiments du hameau dans lequel a été trouvé ce témoignage sont d'ailleurs en voie de réhabilitation, signe que, consciente de la valeur de ces bâtiments, une partie de la population souhaite les faire revivre.

\section{La disparition des ruines, disparition de l'entre-deux}

Depuis quelques années, le nombre de vestiges dans la région baissent d'après nos interlocuteurs. Deux raisons à cela : soit les ruines disparaissent du fait de l'inaction de 
l'homme, elles sont détruites par le temps, soit, au contraire, elles disparaissent sous l'effet de l'action humaine. Dans ce cas-ci, soit, elles disparaissent " par le bas " pourrait-on dire, c'est-à-dire qu'elles sont détruites volontairement ; soit, elles disparaissent "par le haut ", c'est-à-dire sont réhabilitées.

La disparition du lieu de l'entre-deux qu'est la ruine fait souvent apparaitre une nouvelle marge ou peuvent se développer des pratiques alternatives, ou bien des modes de vie originaux. En ce sens, cela produit un nouveau lieu intermédiaire, comme un autre palier dans l'entre-deux. Le plus souvent, les réhabilitations sont le fait de " nouveaux " arrivants, installés ici à partir des années 1970-1980. Certes leur discours n'est pas teinté de la mélancolie qu'ont les personnes originaires de ce territoire, mais les ruines sont toujours des éléments marquants du paysage. Les reprises sont alimentées par une certaine idée du patrimoine, la volonté d'apporter un renouveau, mais aussi de trouver un peu de calme en s'installant dans des lieux à l'écart.

Pour revenir sur quelques une des rencontres que j'ai pu faire, je citerai d'abord le cas de madame L. ; réinstallée il y a une trentaine d'années dans la commune de ses grandsparents, elle a racheté, avec son mari, une maison ruinée. Après l'avoir rénovée, ils ont réhabilité un autre bâtiment qui était sur leur terrain et avaient pour projet, au moment de mon passage, de racheter et rénover une autre ruine située au dessus de chez eux. De même, les A., originaires du bassin-parisien, ont racheté, en 1978, un vieux bâtiment délabré dans un village en ruine surplombant Barcelonnette. Dans le but d'en faire une habitation secondaire, ils ont, petit à petit, reconstruit cette maison et vivent désormais sur place quelques mois par an, un peu à l'écart du monde d'après leurs dires. Cependant, l'absence d'eau courante et la nécessité d'utiliser un groupe électrogène pour se fournir en électricité limitent les séjours aux mois estivaux. Au moment de notre entretien, un second bâtiment avait été acheté dans le hameau, par un nouveau venu, pour être restauré.

Les belges et les hollandais sont nombreux à venir s'installer dans la région. Ainsi, j'ai pu rencontrer monsieur et madame $S$, au Vieux-Bras-d'Asse, village en ruine perché au dessus du village actuel de Bras-d'Asse. Arrivés en 1987, ils ont décidé de s'investir en réhabilitant des bâtiments dans ce village en ruine :

"c'était une occasion, pour rester ici dans le sud. Et puis le climat est attractif pour nous. Et puis on n'aime pas les vacances quand on est assis. On voulait faire des choses. [...] C'est aussi un peu la part écologique de notre esprit : il faut pas toujours changer, changer, changer! On peut respecter et employer ce que les vieux ont fait. II ne faut pas tout renouveler ".

Bras d'Asse est situé non loin de la confluence entre l'Asse et la Durance et a été entièrement déserté au $19^{\text {ème }}$ siècle, tandis qu'un nouveau village était recrée une centaine de mètres plus bas, à proximité de la rivière. Le vieux village en ruine a été tout entier racheté par une association - quelques ruines exceptées, que certains n'ont pas voulu vendre -, et a été petit à petit réhabilité. Aujourd'hui, madame $S$. et son mari sont les deux seuls à s'être s'installés dans le village à l'année tandis d'autres personnes continuent à venir uniquement durant la saison estivale. Ensemble, ils espèrent parvenir à reconstruire tout le village. 
Si les initiatives individuelles ne manquent pas concernant les ruines, si des associations remettent régulièrement en état des bâtiments, il faut souligner que les communes favorisent très rarement les reprises, voire s'y opposent. Ceci notamment parce qu'elles n'ont pas les moyens de payer les raccordements à l'eau et à l'électricité pour les bâtiments isolés qui seraient reconstruits. Dès lors, les conseils généraux favorisent plutôt les installations et les infrastructures dans les bourgs. La reprise d'une maison dégradée dans un lieu isolé, qui était faisable il y a une vingtaine d'année, n'est désormais plus possible.

Les deux cas évoqués ici (reprise des ruines à titre individuel, ou bien réhabilitation par des collectifs) engendrent à mon sens la fin du paysage de l'entre-deux, dans le sens où c'est un bâtiment habité et donc stabilisé qui remplace la ruine.

\section{Conclusion}

Nous sommes ainsi passés, au cours de cette réflexion, par les aspects théoriques autant que pratiques qui permettent de penser le rôle de la ruine dans le paysage, comme un espace de l'entre-deux.

Au moment de refermer cet entre-deux, on entrevoit alors la réponse à la question simple posée en introduction : "qu'est-ce que cela fait que ceci soit là ". On comprend que ce lieu de l'entre-deux, qu'est la ruine, en tant que part du paysage, joue sur les perceptions du territoire, et donc sur la manière de le considérer. Observer ce lieu de l'entre-deux, c'est, en un sens, sentir ce qui n'est plus là dans le paysage, ce qui a changé dans ce territoire, comprendre les transformations en déchiffrant le palimpseste. Cela influe alors directement sur les dynamiques du territoire, dans la mesure où cela attire certaines personnes et en repousse d'autres. Ces marges sont ainsi sans cesse susceptibles d'être réintégrées dans des circuits productifs, même si ces derniers sont eux-mêmes marginaux. Vivre seul, dans une maison réhabilitée au sein d'un village ruiné ; I'on pourrait commencer ici un article sur l'entre-deux, sur l'expérience de vivre dans la marge, mais, comme j'ai tenté de le montrer, c'est ici que s'arrête la mienne.

Cette étude, tirée d'un travail de master, est bien trop lacunaire pour prétendre faire le tour de la question, et j'ai bien conscience de ses faiblesses théoriques et méthodologiques; bien des éléments gagneraient à être creusés et précisés, ce qui n'a pas pu être fait, faute de temps. On peut espérer que d'autres études sur ce thème suivront. 


\section{Bibliographie}

BAZIN G. (1986), "Quelles perspectives pour les agricultures montagnardes ? Exemples du Massif Central et des Alpes du Sud », Etudes et recherches, $n^{\circ} 3,121 \mathrm{p}$.

BETEILLE R. (1974), L'espace humain aveyronnais, la société et l'émigration, Thèse de Doctorat d'Etat de l'Université de Toulouse II.

BETEILLE R. (1981), La France du vide, Paris, LITEC, 252 p.

CERAMAC (2000), Les friches dans le Massif Central, mythes et réalités, Clermont-Ferrand, PUBP, $276 \mathrm{p}$.

CERAMAC (2007), Habiter et vivre dans les campagnes de faible densité, Actes du $2^{\text {ème }}$ colloque franco-espagnol de géographie rurale, Clermont-Ferrand, PUBP, 785 p.

CURNIER J.P. (2009), Montrer l'invisible, écrits sur l'image, Arles, Editions Jacqueline Chambon, Actes Sud, 188 p.

DELSALLE P., (2006), ivre en Franche-Comté au siècle d'Or, XVle-XVIIe siècles, Besançon, Cêtre, 349p.

DE REPARAZ A. (1978), La vie rurale dans les Préalpes de Haute-Provence, Thèse de I'Université d'Aix-Marseille II, EDISUD, 1230 p.

DEUFFIC P. (2005), " La fermeture des paysages dans le Massif Central, regards d'habitants sur une question d'experts ", Cahiers d'économie et sociologie rurales, n75, pp. 76-96.

DESILVEY C., EDENSOR T. (2012), " Reckoning with ruins », Progress in Human Geography, $\mathrm{n}^{\circ} 37$ (4), pp. 465-485.

DONADIEU P., PERIGORD M. (2005), Clés pour le paysage, Collection GéOphrys, Gap - Paris, Ophrys, $368 \mathrm{p}$.

DUBY G., RONCAYOLO M., COURBIN P, et al. (1965), Villages désertés et histoire économique : Xle-XVIIle siècles, Paris, EPHE, 619 p.

FABRE E., VERNET C. (2006), "Evolution de l'occupation du sol dans les Alpes de HauteProvence ", Méditerranée, $n^{\circ} 3-4$, pp. 35-42.

FLAUBERT G. (1881), réed. 1950, Bouvard et Pécuchet, Folio Classique, Paris, Gallimard.

FREMONT A. (1979), " Marginalité et espace vécu », in Beauchard J. (dir) Identités collectives et travail social, Toulouse, Privat, pp. 99-113

GENDRAT-CLAUDEL A. (2007), Le paysage, fenêtre ouverte sur le roman, Paris, PUPS, p. 69

JANIN C. (2008) "Les friches, espaces en marge ou marges de manœuvre pour l'aménagement des territoires ? ", Annales de géographie, n663, p. 62-81. 
JEAN Y., PERIGORD M. (2009), Géographie rurale, la ruralité en France, Paris, Armand Colin, $126 \mathrm{p}$.

LACROIX S. (2007), Ce que nous disent les ruines, la fonction critique des ruines, Paris, L'Harmattan, 318 p.

LE FLOCH S., DEVANNE A. S., DEFFONTAINES J.P. (2005), " La fermeture du paysage : Au-delà du phénomène, petite chronique d'une construction sociale ", L'espace Géographique, $n^{\circ} 1$, pp. 49-64.

LUGINBUHL Y. (2007), "Pour un paysage du paysage », Economie rurale, n²97-298, janvieravril, pp 23-40.

LUSSAULT M., LEVY J. (dir) (2003), Dictionnaire de la géographie, Paris, Belin, 381 p.

MADELINE P. (2006) "Les constructions agricoles dans les campagnes françaises. Héritages et dynamiques actuelles d'évolution ", Histoire et sociétés rurales, 2, n²6, p. 53-93.

MERLIN P. (1969), La dépopulation des plateaux de Haute-Provence, Paris, La documentation française, $182 \mathrm{p}$.

MOUSTIER P. (2006) «Déprise agricole et mutations paysagères depuis 1850 dans le Champsaur et le Valgaudemar (Hautes-Alpes) », Méditerranée, n¹07, pp. 43-51.

MICHEL D'ANNOVILLE N., DE LEEUW M. (2008), Les Hautes Terres de Provence, itinérances médiévales, Saint-Michel l'Observatoire, Les Hautes Terres de Provence, 224p.

RIEGL A. (1903), Le culte moderne des monuments, trad. fr. D. Wieczorek, 1984, Paris, Editions du Seuil, 122 p.

ROGER A. (dir) (1995), La théorie du paysage en France (1974-1994), Seyssel, Champs Vallon, $463 \mathrm{p}$.

RONCAYOLO M. (1965), "Géographie et villages désertés ", Annales. Economie, sociétés, civilisations, vol 20, $n^{\circ} 2$, pp 218-242.

SCLAFERT T. (1959), Cultures en Haute-Provence : déboisements et pâturages au Moyen Age, Paris, SEVPEN, EPHE, $272 \mathrm{p}$.

SIMMEL G. (1912), Mélanges de philosophie relativiste. Contribution à la culture philosophique, trad. Guillain A., 1912, Paris, Bibliothèque de philosophie contemporaine, Librairie Félix Alcan, 268 p.

VESCHAMBRE V. (2008), Traces et mémoires urbaines: enjeux sociaux de la patrimonialisation et de la démolition, Rennes, PUR.

VIDAL-FONTILLE C. (1988), La population des Alpes du Sud de 1860 à nos jours, Aix-enProvence, Ophrys, 662 p. 
VIDAL DE LA BLACHE P. (1911), " De l'interprétation géographique des paysages », Neuvième Congrès International de Géographie, Compte rendu des travaux du Congrès, Genève, Société Générale d'Imprimerie, pp. 59-64.

ZWICKERT F. (1961), L'avenir des terres et villages en voie d'abandon dans les Basses-Alpes, Mémoire de Stage, ENA, $25 \mathrm{p}$. 